\title{
Automatic Guided Vehicle Global Path Planning Considering Multi-objective Optimization and Speed Control
}

\author{
Jing Song* \\ College of Port and Shipping Management, Guangzhou Maritime University, Guangzhou 510725, China
}

(Received December 31, 2020; accepted April 12, 2021)

Keywords: multi-objective optimization, global path planning, inductive steering algorithm, quantum particle swarm optimization algorithm, automatic guided vehicle

Over the past few decades, as the main tool of intelligent material transportation, automatic guided vehicles (AGVs) have been widely used in modern production systems, logistics, transportation, industry, and commerce to further improve productivity, reduce labor costs, raise energy efficiency, and enhance safety. Path planning is a key issue in the field of AGVs to ensure that they do not collide with obstacles during movement and reach the destination as fast as possible to complete the assigned task. We propose two different and crucial operating environments in this paper. More specifically, in a static environment, a multi-objective mathematical model is established with the shortest path and the maximum smoothness, and the improved Levy random quantum particle swarm optimization (LRQPSO) algorithm is used to solve the proposed model and screen the AGV's driving path. In a dynamic environment, an inductive steering algorithm (ISA) that considers the movement of obstacles is proposed to make the AGV avoid obstacles rationally. By combining the steering characteristics of the two environments, AGV speed control rules are set and applied to the steering process in complex environments to ensure that the AGV can travel more smoothly and quickly. Simulation results show that the proposed method can ensure the obstacle avoidance and flexible steering of an AGV, and improve the driving speed and work efficiency in the two environments. In addition, compared with the conventional algorithm, the smoothness, operation speed, and work efficiency of the AGV are significantly increased using the improved LRQPSO algorithm and ISA.

\section{Introduction}

The extensive use of automatic guided vehicles (AGVs) in industrial warehouses has greatly improved the efficiency and accuracy of product circulation and distribution, and enhanced the flexibility of the production system. In addition, there is growing interest in AGV path planning to help improve the work efficiency and flexibility of foundations. Therefore, both accurate and effective path planning are a research focus in this paper. The path planning problem of how to avoid obstacles in the most rational way to complete tasks was proposed by Dorigo and

*Corresponding author: e-mail: 84864809@qq.com

https://doi.org/10.18494/SAM.2021.3280 
Gambardella ${ }^{(1)}$ in the 1990s. Because the path optimization problem is NP-complete in nature, many heuristic algorithms of path planning have appeared successively over the past decade. ${ }^{(2)}$

The environment map of the AGV workspace can be static or dynamic. Thus, the algorithm performance of the path planning problem in a static environment is lower than that of dynamic programming. In contrast, the obstacles in the AGV workspace move in real time, which makes the path planning problem in a dynamic environment more complicated. To achieve a reliable path, the AGV must have real-time access to environmental information and be able to make rapid changes to a previously planned path. ${ }^{(3)}$ Compared with AGV models guided by an ultrasonic wave, laser, or magnetic tape, an AGV guided by a visual sensor has the advantages of a fast response, low cost, and the acquisition of a large amount of information. Visual guidance technology has been widely used in engineering because of its high information dimension and flexibility in guidance. Therefore, path planning of an AGV based on visual guidance has become an important research hotspot.

With the proliferation of big data, the Internet, and artificial intelligence (AI), the AGV path planning problem can be formulated as a novel category of a constrained optimization problem. In addition, most scholars have studied the design optimization of AGV path planning algorithms, which can be further divided into traditional optimization algorithms and computational intelligent algorithms. The traditional optimization algorithms mainly include the $\mathrm{A}^{*}$ algorithm, ${ }^{(4)} \mathrm{D}^{*}$ algorithm, ${ }^{(5)}$ artificial potential field algorithm, ${ }^{(6)}$ unit decomposition method, and rapidly exploring random trees (RERT). ${ }^{(7)}$ Path planning has been widely viewed as an NP-hard problem. With the increasing complexity and dynamics of environment maps, most traditional algorithms have the defects of a high solving cost, a large probability of trapping at local extrema, and low solving efficiency. To overcome these issues, many scholars have extensively studied the application of various intelligent optimization algorithms in the AGV path planning domain. ${ }^{(8)}$ Intelligent solving algorithms primarily consist of the genetic algorithm $(\mathrm{GA}),{ }^{(9)}$ flower pollination algorithm (FPA), ${ }^{(10)}$ grey Wolf optimization (GWO) algorithm, ${ }^{(11)}$ imperialist competition algorithm (ICA), ${ }^{(12)}$ teaching-learning-based optimization (TLBO) algorithm, ${ }^{(13)}$ and particle swarm optimization (PSO) algorithm. ${ }^{(14)}$ Compared with traditional solving algorithms, intelligent algorithms have better robustness in solving path planning problems. ${ }^{(15)}$ Owing to its robustness, the GA has been widely used in numerous optimization fields, such as cloud manufacturing service combination optimization, ${ }^{(16)}$ the travel agent problem, AGVs, and integrated scheduling of processing operations. ${ }^{(17)}$

In view of the low efficiency of the PSO algorithm and the problem that path optimization algorithms often get trapped at local optima, we propose an improved Levy random quantum PSO (LRQPSO) algorithm and an induction steering algorithm (ISA) for static and dynamic environments, respectively. This paper not only considers the shortest path and maximum smoothness but also the speed limit to study AGV path planning. In particular, we apply the LRQPSO algorithm to successfully resolve the multi-objective mathematical model to generate the optimal path in a static environment. The ISA is applied to the steering behavior in static and dynamic environments to make the AGV performance more flexible. At the same time, in accordance with a kinematic model such as that of an automobile mobile robot, steering speed rules are formulated for an $\mathrm{AGV}$ to ensure its smooth and fast operation and improve work efficiency. 


\section{Materials and Methods}

There have been many studies on intelligent algorithms to cope with the dynamic path optimization problem of AGVs. Algorithms include the bacterial potential field (BPF) algorithm, which combines the artificial potential field (APF) algorithm and the bacterial evolutionary algorithm (BEA), ${ }^{(18)}$ which are broadly applied to deal with the static and dynamic path optimization problem for mobile robots. The BPF algorithm optimizes the coefficients of attraction and repulsion by employing the BEA and effectively avoids the shortcoming of trapping at local extrema of the traditional APF algorithm. Simulation experiments have proved that the BPF algorithm is valid in both static and dynamic environments, but its downside is the simple environment map and obstacle movement. In the solution of the optimization problem using the traditional GA, the mutation operator randomly generates a new gene to replace the original one with a certain probability, which results in the low probability of a better solution. To overcome this problem, Tuncer and Yildirim proposed a new mutation operator that determines the candidate point set in the free space in the neighborhood of the gene to be mutated, then replaces the original gene with a randomly selected point from the candidate point set for mutation, which is helpful for improving the probability of mutation to generate good solutions. ${ }^{(19)}$ An improved GA based on the improved mutation operator has been proposed, which can solve the dynamic path planning problem of a mobile robot, proving the effectiveness of the improved strategy. However, the selection of the mutation point has no heuristic information, which leads to the low probability of a mutation generating good solutions. Zhu and Peng proposed a new mutation operator based on the mountain-climbing algorithm to effectively avoid the problem of the traditional GA of the solution for the path optimization problem. ${ }^{(20)}$ The viewable algorithm is improved to model the environment map, which effectively improves the security of the planned path. To improve the GA optimization efficiency, the PSO algorithm was applied to refresh the population after each genetic operation. Simulations and experiments were also performed to evaluate the effectiveness of the modified GA in resolving the dynamic path planning problem of mobile robots. However, the disadvantages of the modified GA are a simple environment map, simple obstacle movement, and few dynamic obstacles. Das et al. proposed an improved gravity search algorithm (GSA) based on PSO, and solved the path planning problem of multiple mobile robots. Simulation-based and practical experiments proved the effectiveness of this algorithm. However, a disadvantage of the improved GSA is that the obstacles in the workspace are static. ${ }^{(21)}$ An improved initial solution generation algorithm based on the visual space method was proposed, and a new mutation operator to avoid premature convergence was proposed, on the basis of which an improved GA was presented. ${ }^{(22)}$ It was proven experimentally that the improved GA solved the static and dynamic path planning problems efficiently, although a disadvantage was that there could be only one dynamic obstacle in the dynamic environment. Li et al. proposed an algorithm based on the PSO algorithm and the Legendre pseudospectra method (LPM) called the PSO-LMP algorithm, which can be divided into the following two stages to solve the dynamic path planning problem. The first stage uses the advantages of the PSO algorithm to conduct a global search. ${ }^{(23)}$ After reaching the termination conditions of the first stage, the algorithm enters the second stage of the search, which uses the LPM algorithm. A 
simulation-based experiment proved the effectiveness of the PSO-LPM algorithm for solving the dynamic path planning problem. Liao et al. proposed an improved GWO algorithm and GA for solving the static path planning problem of an $\mathrm{AGV}$, then designed a path smooth processing algorithm, and finally established a dynamic path planning model. Importantly, a simulation platform for AGV static and dynamic path planning was developed on the basis of the MATLAB GUI development tool. The application of the improved GA to solving a variety of AGV dynamic path planning problems under an environment map proved the effectiveness of the improved algorithm. ${ }^{(24)}$ Alajmi and Almeshal optimized the trajectory of mobile robots by using the quantum particle swarm optimization (QPSO) algorithm, which has a high performance, enhances the ergodic property of the particle space, and improves the search ability. ${ }^{(25)}$

All the above algorithms demonstrated their applicability in the research field after improvement, and the performances of the algorithms themselves were also improved. However, many path planning solutions ignored the motion state caused by the mechanical properties of robots. Owing to the limitations of moving robots, they usually move at a low speed during operation, making it difficult to improve work efficiency.

\section{Problem Formulation and Modeling}

\subsection{Problem formulation}

In a workspace, the AGV moves from the source point to the destination point to complete the transportation task. It is necessary that the AGV can avoid static obstacles while moving, and the moving path meets the requirements of multi-objective constraints, i.e., the shortest path and the minimum sum of the steering angles. Assuming that the environmental information of the AGV workspace is known, the AGV workspace is defined as the physical space represented by $R$ $\times R$, the obstacle mapping in the workspace has an obstacle space $C_{o b s}$, and the free space without collision and movement between the AGV and the obstacles is denoted as $C_{\text {free }}$. First, the multi-objective function model is established for spatial searches, and then the most promising solution is applied to the proposed optimization model. Finally, the Pareto solution set that conforms to the best constraint path is screened. To complete the task of AGV movement, two objective constraints must be met: one is the shortest moving path and the other is the maximum smoothness of the path, namely, the minimum sum of the steering angles. The results when these two factors are regarded as the target constraints are as follows: the shorter the path, the shorter the moving time and the faster the AGV can reach the destination point. On the other hand, a smoother path can reduce the mechanical wear of the AGV shaft wheels and decrease energy consumption. The shortest path objective function is

$$
\Phi_{1}\left(n(t a), P_{i}\left(x_{i}, y_{i}\right), P_{i+1}\left(x_{i+1}, y_{i+1}\right)\right)=\min \sum_{i=1}^{n(t a)} \sqrt{\left(x_{i+1}-x_{i}\right)^{2}+\left(y_{i+1}-y_{i}\right)^{2}}
$$

where $\Phi_{1}\left(n(t a), P_{i}\left(x_{i}, y_{i}\right), P_{i+1}\left(x_{i+1}, y_{i+1}\right)\right), n(t a), P_{i}\left(x_{i}, y_{i}\right)$, and $P_{i+1}\left(x_{i+1}, y_{i+1}\right)$ define the shortest path objective function, the total number of the steering angles, and the coordinates of the initial 
point and destination, respectively. The maximum smoothness objective function is defined as

$$
\begin{aligned}
& \Phi_{2}\left(n(t a), P_{i}\left(x_{i}, y_{i}\right), P_{i+1}\left(x_{i+1}, y_{i+1}\right)\right) \\
& =\min \sum_{i=1}^{n(t a)}\left(P_{i-1}\left(x_{i-1}, y_{i-1}\right), P_{i}\left(x_{i}, y_{i}\right), P_{i+1}\left(x_{i+1}, y_{i+1}\right)\right) \cdot\left|\arctan \left(\frac{y_{i+1}-y_{i}}{x_{i+1}-x_{i}}\right)-\arctan \left(\frac{y_{i}-y_{i-1}}{x_{i}-x_{i-1}}\right)\right| .
\end{aligned}
$$

\subsection{Multi-objective optimization of Pareto optimal solution}

The path planning solution in this paper also involves two factors, the shortest path and the maximum smoothness, so it can be defined as a multi-objective problem (MOP). For further details of the MOP model, including the Pareto disaggregation, the Pareto optimal solutions as well as the solution set, and the non-inferior solution, please refer to the literature. ${ }^{(22)}$ Using the proposed LRQPSO algorithm to solve the MOP model will produce multiple non-inferior solutions due to the lack of direction of the attributed particle movement. However, the AGV must be able to choose a path to move at any time. To reduce the effect of the randomness of people's movement on the LRQPSO algorithm, the Pareto solution set is evaluated in detail. A promising method proposed in the literature is introduced to describe the crowding distance between non-inferior solutions, ${ }^{(23)}$ and the crowding distance $L_{i}$ is defined as

$$
L_{i}=\sum_{n=1}^{N}\left(\frac{f_{n}^{i+1}-f_{n}^{i-1}}{f_{n}^{\max }-f_{n}^{\min }}\right), i=2,3, \ldots, k-1 .
$$

Here, $i$ is the total number of non-inferior solutions, parameters $f_{n}^{\max }$ and $f_{n}^{\min }$ describe the maximum and minimum values in the Pareto solution set for the $n$th objective function, respectively, and $N$ is the total number of objective functions. The larger the crowding distance, the lower the probability of crowding among individuals, indicating that the better the diversity of the population, the greater the probability that the population will be retained during screening.

\subsection{Velocity planning in complex environments}

The structure of the AGV is similar to that of a universal vehicle. AGV guidance methods include electromagnetic induction guidance, laser guidance, and visual guidance. Owing to the rapid development and maturation of AGV technology, various types of $\mathrm{CCD}$ cameras and sensors can be deployed on AGVs. The on-board computer set in the AGV analyzes the path environment in complex environments, determines the current position, and moves to the next step. We can achieve the following attributes: the four wheels can roll and rotate without sliding, and the fixed rear wheels are parallel to the body. The front wheels can rotate freely. Figure 1 depicts the structure of the steering process in detail. The two wheels must be parallel or satisfy the Ackerman steering geometry. 


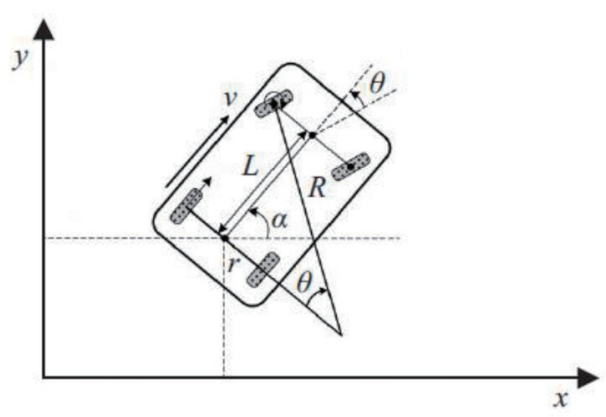

Fig. 1. Detailed architecture of AGV.

The motion model of the AGV is as follows:

$$
\begin{aligned}
& \left\{\begin{array}{l}
x=v \sin a, \\
y=v \cos a, \\
a=\frac{v \tan \theta}{L}
\end{array}\right. \\
& \text { s.t. }\left\{\begin{array}{l}
x=v \sin a-y \cos a, \\
y=x \sin (\theta+a)-y \cos (\theta+a)-L a \cos \theta,
\end{array}\right.
\end{aligned}
$$

where $x$ and $y$ are the horizontal and vertical velocity components, respectively, $\theta$ is the steering wheel angle, $a$ is the angle between the vehicle body and the $X$ axis, and $L$ is the wheelbase. According to the above constraints, the direction of motion of the AGV is parallel to the vehicle body direction, and there is a minimum radius limiting the speed. The motion constraints of AGV steering are as follows:

$$
\begin{aligned}
& \xi_{\min } \geq m \cdot \frac{v_{\max }^{2}}{R}, k=\frac{1}{R}, \\
& v_{\max }=\sqrt{u g \sqrt{\left(1+L^{2} \cdot k^{2}\right)} \sqrt{R^{2}-L^{2}}}, \\
& v_{i} \leq v_{\max },
\end{aligned}
$$

where $k, R, v_{\max }, u, g, v_{i}$, and $m$ are the curvature, turning radius, maximum steering speed, static friction coefficient, gravitational acceleration, running velocity, and vehicle mass, respectively.

\section{Path Planning in Static Environment}

\subsection{Prior PSO algorithm}

The prior PSO algorithm, inspired by the social behavior of bird foraging, is a randomized optimized intelligent technology for simulating the self-cognition, cooperation, and information 
exchange among individuals with the goal of finding the optimal solution. Assuming that the search space of each particle lies in the population of $\Theta$, the position presentation vector and the velocity measure vector of the $i$ th particle are respectively expressed as $l o c_{i}=\left(\operatorname{loc}_{i}(1), \operatorname{loc}_{i}(2), \ldots, \operatorname{loc}_{i}(n)\right)$ and $v^{2} l_{i}=\left(\operatorname{vel}_{i}(1), \operatorname{vel}_{i}(2), \ldots, v^{2} l_{i}(n)\right)$, where $n$ is the dimension of the search space. The $i$ th particle velocity and position can be updated from time $t$ to time $t+1$ in any dimension $d$ $(d=1,2, \ldots, n)$ as

$$
\begin{aligned}
& \operatorname{vel}_{i d}(t+1)=\alpha \cdot v e l_{i d}(t)+\beta \cdot \lambda_{1}\left(\operatorname{pbest}_{i d}(t)-\operatorname{loc}_{i d}(t)\right)+\gamma \cdot \lambda_{2}\left(\operatorname{gbest}_{d}(t)-\operatorname{loc}_{i d}(t)\right), \\
& \operatorname{loc}_{i d}(t+1)=\operatorname{vel}_{i d}(t)+\operatorname{loc}_{i d}(t),
\end{aligned}
$$

where $\alpha$ is the inertial coefficient, $\beta$ and $\gamma$ are the individual learning factor and social learning factor, respectively, and parameters $\lambda_{1}$ and $\lambda_{2}$ are random numbers in the range $[0,1]$.

\subsection{Improved LRQPSO}

The formulated LRQPSO algorithm applies the idea of quantum motion to particle search behavior, and its speed and position state are updated using Eq. (7). It was previously shown that the introduction of Levy flight into the PSO approach can make the search escape from local optima and improve the algorithm performance. ${ }^{(14,15)}$ An orderly particle search that does not repeat itself can efficiently reduce the waste of the search process. The random learning mechanism of particles can enhance the categories of the defined population paradigm and prevent premature convergence, which is similar to the mutation in the GA. This learning mechanism enables particles to search in an optimal orientation after optimization and ensures the ability to generate global optimal solutions. The proposed algorithm can avoid higher computation complexity to some extent. Therefore, the improved algorithm is defined as the LRQPSO approach with its state update given by Eq. (7). Algorithm 1 describes the pseudocode of the proposed LRQPSO algorithm.

$$
\begin{aligned}
& \operatorname{vel}_{i d}(t+1)=\frac{c_{1} r_{1} \cdot p_{i}(t)+c_{2} r_{2} \cdot p_{g}(t)+c_{3} r_{3} \cdot p_{r}(t)}{c_{1} r_{1}+c_{2} r_{2}+c_{3} r_{3}}, \\
& \operatorname{loc}_{i d}(t+1)=\operatorname{Levy}\left(\beta_{i}\right)\left(l o c_{i d}(t)-p_{g}(t)\right) \cdot w\left|m_{\text {best }}-l o c_{i d}(t)\right| \ln \frac{1}{u}, \\
& m_{\text {best }}=\frac{1}{M} \sum_{i=1}^{M} p_{g}(t), \\
& w=0.5+0.5 \times \frac{\max \text { gen }- \text { iter }}{\max \text { gen }}, \\
& \operatorname{Levy}\left(\beta_{i}\right) \sim \frac{\lambda_{i}}{\left|\gamma_{i}\right|^{\frac{1}{\beta_{i}}}} .
\end{aligned}
$$


Algorithm 1.

Pseudocode of the proposed LRQPSO algorithm.

Initialization: $Q$, max gen, $c_{1}, c_{2}, c_{3}$

Calculation of fitness value: The particle form is transformed into the quantum particle form, and the Levy flight and particle random learning mechanism are introduced.

Iteration: The non-inferior solutions are stored in $Q$ and compared with other non-inferior solutions to generate a new non-inferior solution set.

Computation of crowding distance: The non-inferior solution with the smallest crowding distance is eliminated and then a new non-inferior solution is introduced for cyclic calculation.

If the result satisfies the terminal condition, then

output $G_{\text {best }}$ and fitness value

Else Return step 3

End

\section{Path Planning in Dynamic Environment}

AGVs moving along the planning path in a static environment can be calculated as above. However, when there are multiple AGVs in the same environment that is changing dynamically, static path planning cannot achieve feasibility in many cases. In such a case, secondary path planning based on the ISA approach is carried out, as discussed in this section. Importantly, time delay theory is commonly used as a metric constraint so that multiple AGVs avoid each other. Although this method can ensure the avoidance of a small number of AGVs, the calculation will not be able to handle a larger number of AGVs because of the nonideal solution effect. Toward this end, we propose a novel method to avoid collision in a dynamic environment that uses current induction technology, namely, the ISA approach, to generate several non-intersecting curves uniformly at the induction starting point, calculate the curvature of each curve, and select the curve with the least curvature and no collision as the driving path. The main features of this algorithm are that the smaller the curvature, the smaller the turning radius, which leads to the shorter path and the greater smoothness.

\subsection{AGVs meeting fixed obstacles}

Because the network map of obstacles is not updated in a model with events at discrete times, the situation that goods (obstacles) or staff temporarily appear in the AGV workspace may occur. Toward this end, the proposed ISA approach is employed to ensure avoidance only if there is an AGV. There are two cases of unknown fixed obstacles in this paradigm, as shown in Fig. 2.

It is necessary for a marked AGV to avoid collision or slip caused by instantaneous inertia. To reduce the probability of this occurring, the path smoothness, which has a similar definition to the curvature, is then selected as the basis for steering. The smaller the curvature, the greater the smoothness, resulting in shorter paths, smaller changes in velocity, and safer movement. The steering mode of Fig. 2(b) is an extension of that in Fig. 2(a). From the result fed back from the induction device, the AGV processor can generate multiple alternative routes, among which the collision-free path with the least curvature is selected. The first and second derivatives of the curve are continuous, the trajectory in the form of a curve is introduced into the coordinate system, and the curve equation is orthogonally decomposed. The trajectory equation is 


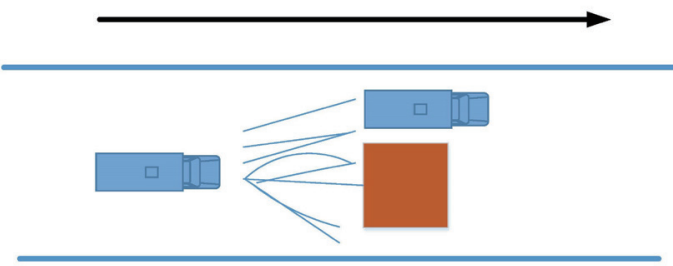

(a)

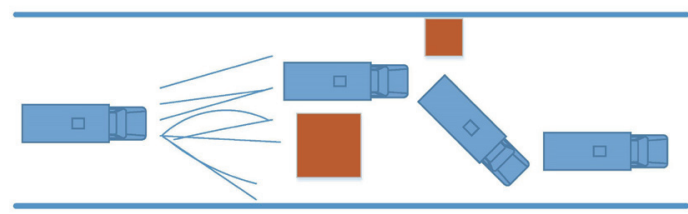

(b)

Fig. 2. (Color online) Cases of AGV's obstacle avoidance.

$$
y_{0}=\alpha x_{0}^{3}+\beta x_{0}^{2}+\gamma x_{0}+\rho \text {. }
$$

Therefore, the curve equation can be further decomposed orthogonally to the following:

$$
\begin{gathered}
\left\{\begin{array}{l}
x_{0}=\alpha_{x} x^{3}+\beta_{x} x^{2}+\gamma_{x} x+\rho, \\
y_{0}=\alpha_{y} y^{3}+\beta_{y} y^{2}+\gamma_{y} y+\rho,
\end{array}\right. \\
\theta_{\text {min }}=\arctan \frac{d y}{d x}, \\
\text { s.t. } \quad k_{\min }=\frac{x_{0}^{\prime} y_{0}^{\prime \prime}-x_{0}^{\prime \prime} y_{0}^{\prime}}{\sqrt{\left(x_{0}^{\prime}+y_{0}^{\prime}\right)^{3}}}, \\
\sqrt{\left(x_{0}-x^{*}\right)^{2}+\left(y_{0}-y^{*}\right)^{2}} \geq R .
\end{gathered}
$$

\subsection{Multiple AGVs meeting in opposite directions}

When multiple AGVs move in opposite directions, both $\mathrm{AGV}_{i}$ and $\mathrm{AGV}_{j}$ determine the steering direction according to the distance between the center line of $\mathrm{AGV}_{i}$ and the edge of $\mathrm{AGV}_{j}$ after mutual inductance, which is assisted to complete the steering at half of the sensing distance. The movement mode of the AGV should satisfy the following constraints:

$$
\begin{aligned}
& L_{i j}=\sqrt{\left(y_{j}^{1}-y_{i}^{1}\right)^{2}+\left(x_{j}^{1}-x_{i}^{1}\right)^{2}} \geq 2 R, \\
& \theta_{\min }=\arctan \frac{y_{j}^{1}-y_{i}^{0}}{x_{j}^{1}-x_{i}^{0}} .
\end{aligned}
$$




\subsection{Multiple AGVs meeting in the same direction}

In this case, multiple AGVs are driving in the same direction in the workspace. When they encounter unknown obstacles in front of them, two avoidance schemes are implemented to avoid collision, namely, overtaking or following.

\section{Results and Discussion}

In this section, multi-objective path optimization and algorithm performance evaluation are first performed in a static environment. In the experimental situation, how to avoid obstacles in a dynamic environment is described, the ISA and shortest distance algorithm (SDA) are then compared, and finally the acceleration and velocity changes of an AGV under the conditions of these two algorithms are finally given. The simulated AGV is used for path planning in a $100 \times 100 \mathrm{~m}$ warehouse with starting point $\mathrm{S}(5,10)$ and end point $\mathrm{T}(65,85)$, and there are six obstacles. First, the proposed LRQPSO algorithm can achieve the optimal solution in resolving the multi-objective model, and the solution set conforming to the constraint is generated as the initial path. Then, the LRQPSO algorithm is compared with the commonly used NSGA-II and SPEA2 algorithms, and further new paths are planned. Finally, the crowding distances of the three algorithms are calculated and compared to highlight the effectiveness of the proposed LRQPSO algorithm in solving the MOPs. The parameters used in the simulation are shown in Table 1.

\subsection{Path planning in static environment}

In the LRQPSO algorithm, the particle position is formulated in the form of coordinates. The search essentially involves changes in the position caused by updating the particle velocity. The fitness values of the algorithm solutions are quantized as the sum of the shortest paths and the sum of the tangential values of the minimum steering angle, and the parallel search method is further adopted to find numerous Pareto front solutions. Then, the parameters of the NSGA-II algorithm are set as follows: the population size is 300 , the maximum number of iterations is 1000 , the crossover probability is 0.8 , the mutation probability is 0.2 , and the number of outstanding individuals retained from the previous generation is six. This method also improves

Table 1

Parameters used in simulation.

\begin{tabular}{lcc}
\hline Symbol & Description & Value \\
\hline max gen & Maximum number of iterations & 1000 \\
$M$ & Population size & 300 \\
$c_{1}, c_{2}, c_{3}$ & Random learning factor & $1.4995,1.4995,2$ \\
$Q$ & Size of external storage & 5 \\
$R$ & Sensing distance & 4 \\
$v_{i}$ & Vehicle velocity & $4 \mathrm{~m} / \mathrm{s}$ \\
$v_{\max }$ & Maximum steering velocity & $0.2 \mathrm{~m} / \mathrm{s}$ \\
\hline
\end{tabular}


the effective convergence rate of the outstanding individuals, and the solution algorithm is better than the initial GA. In this paper, although the number of iterations of the algorithm and the population are larger, we can obtain a shorter path distance and minimum smoothness, and inverse inflection points never appear. Although the time complexity of the proposed LRQPSO algorithm is larger, the accuracy of the solution is greatly increased. The parameter settings and the computing environment of the SPEA2 algorithm are the same as those of the NSGA-II algorithm, and each algorithm is performed five times. The generated objective results are shown in Fig. 3, where PL is the path length and SA is the steering angle.

\subsection{Path planning in dynamic environment}

A dynamic environment refers to a moving space with moving AGVs and staff in addition to known static obstacles. Because a sub-cycle dynamic steering algorithm is embedded into the LRQPSO algorithm, the dynamic steering algorithm is first employed when faced with dynamic obstacles, followed by the complete LRQPSO algorithm. AGVs and dynamic obstacles are mainly discussed here. When multiple AGVs meet, we adopt the proposed ISA and the SDA calculation path in the literature to avoid obstacles. ${ }^{(17)}$ The moving distance, curvature, and other parameters for the ISA and SDA are shown in Table 2, where $L$ is the moving distance, $\theta$ is the path curvature, $a$ is the acceleration change, and $b$ is the velocity change. As can be seen from Table 2, compared with the SDA, the proposed ISA has a shorter moving distance, a smoother path, and can provide a more secure and energy-saving operation mode.

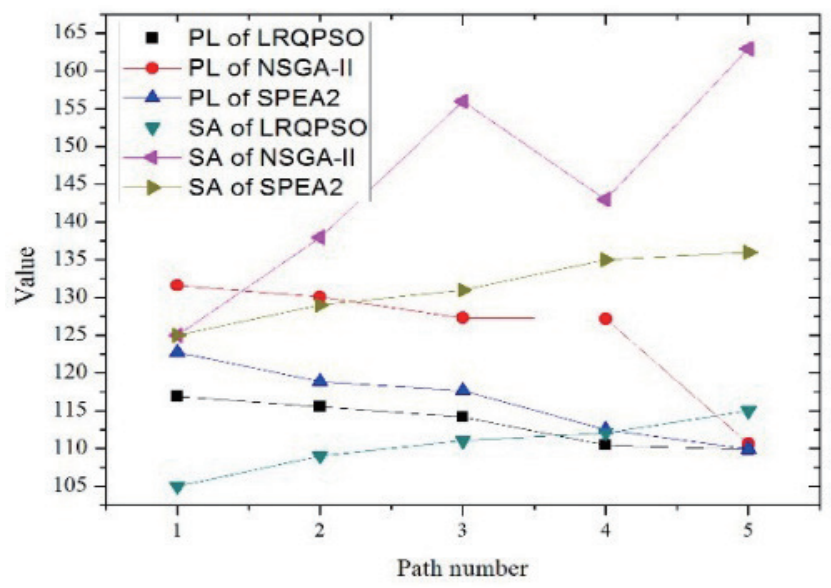

Fig. 3. (Color online) Path and related information for LRQPSO, NSGA-II, and SPEA2 algorithms.

Table 2

Comparison of ISA- and SDA-related parameters.

\begin{tabular}{lcccc}
\hline Algorithm & $L$ & $\theta$ & $a$ & $b$ \\
\hline SDA & 4.81 & 0.11 & $(-0.37,0.37)$ & 0.35 \\
ISA & 4.18 & 0.21 & $(-0.30,0.30)$ & 0.05 \\
\hline
\end{tabular}




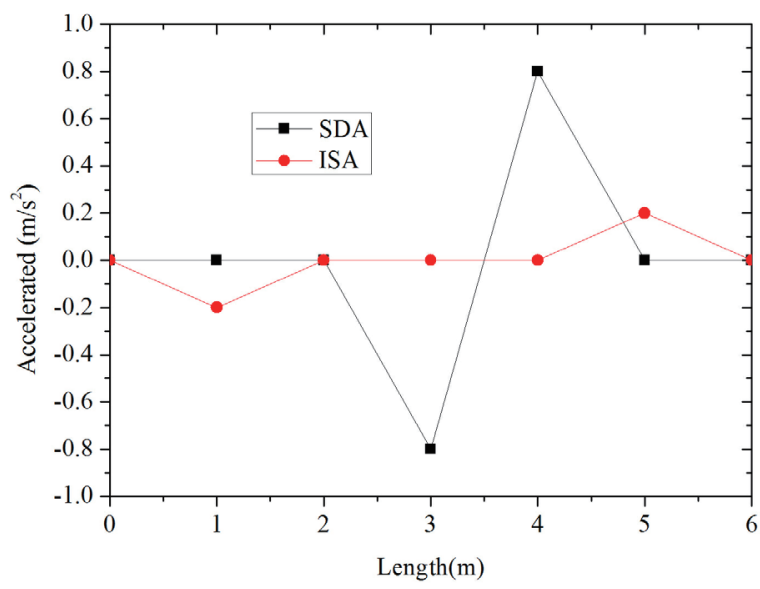

Fig. 4. (Color online) Acceleration/distance.

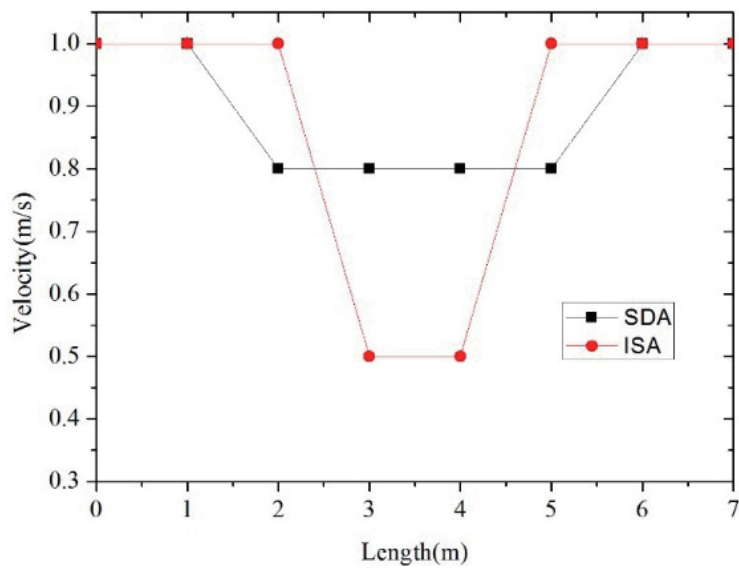

Fig. 5. (Color online) Velocity/distance variation.

To better understand the motion mechanism of the ISA and SDA, the variation in their relationships between acceleration/velocity and distance is used to explain the two algorithms. According to the motion formula of classical physics, the greater the acceleration, the greater the velocity change and the lower the stability of AGV motion. The ISA can better control the AGV's moving acceleration, reduce the speed change, and make the AGV run more smoothly and quickly, as shown in Fig. 4.

After determining the starting position, the ISA is superior to the SDA in terms of moving distance and path smoothness. It can be seen from Figs. 4 and 5 that the acceleration and velocity changes of the ISA algorithm are greater. The smaller the acceleration change, the less likely the cargo on the AGV will slip and the safer it will be at higher speeds.

\section{Conclusions}

We have proposed a global path planning method for use in static and dynamic environments via a multi-objective optimization and speed control method. Comparison of the LRQPSO algorithm with the prior NSGA-II and SPEA2 algorithms shows that the former algorithm can obtain a higher-quality frontier solution when solving the application problem of the multiobjective mathematical model in a static environment. The ISA proposed in this paper is applied to avoid obstacles in a dynamic environment, which can reduce the changes in the velocity and acceleration during AGV steering. Our results show that the proposed algorithm not only can make an AGV run more stably and more in line with actual work requirements, but also has good applicability to dynamic avoidance behavior.

\section{Acknowledgments}

This work was supported by Guangzhou Maritime University Innovation and Strength Project in 2017-Logistics Engineering Key Major Construction. 


\section{References}

1 M. Dorigo and L. M. Gambardella: IEEE Trans. Evol. Comput. 1 (1997) 53. https://doi.org/10.1109/4235.585892

2 H. Ahmead, S. Qure, and A. Yasar: Rob. Auton. Syst. 68 (2015) 1. https://doi.org/10.1016/j.robot.2015.02.007

3 D. Bechtsis, N. Tsolakis, D. Vlachos, E. Iakovou: J. Cleaner Prod. 142 (2017) 3970. https://doi.org/10.1016/j. jclepro.2016.10.057

4 A. Ammar, H. Bennaceur, I. Chaari, A. Koubâa, and M. Alajlan: Soft Comput. 20 (2016) 4149. https://doi. org/10.1007/s00500-015-1750-1

5 I. Maurović, M. Seder, K. Lenac, and I. Petrović: IEEE Trans. Syst. Man Cybern.: Syst. 48 (2018) 1321. https:// doi.org/10.1109/TSMC.2017.2668603

6 H. Min, Y. Lin Y, S. Wang, F. Wu, and X. Shen: Adv. Mech. Eng. 7 (2015) 1. https://doi. org/10.1177/1687814015619276

7 I. Noreen, A. Khan A, H. Ryu, N. L. Doh, and Z. Habib: Intel. Serv. Rob. 11 (2018) 41. https://doi.org/10.1007/ s11370-017-0236-7

8 X. Zhong, J. Tian, H. Hu, and X. Peng: J. Intell. Rob. Syst. 99 (2020) 65. https://doi.org/10.1007/s10846-01901112-Z

9 A. Bakdi, A. Hentout, H. Boutami, A. Maoudj, O. Hachour, and B. Bouzouia: Rob. Auton. Syst. 89 (2017) 95. https://doi.org/10.1016/j.robot.2016.12.008

10 Y. Zhou and R. Wang: Int. J. Pattern Recognit. Artif. Intell. 30 (2016) 1659010. https://doi.org/10.1142/ S0218001416590102

11 S. Zhang, Y. Zhou, Z. Li, and W. Pan: Adv. Eng. Software 99 (2016) 121. https://doi.org/10.1016/j. advengsoft.2016.05.015

12 H. Duan and L. Huang: Neurocomputing 125 (2014) 166. https://doi.org/10.1016/j.neucom.2012.09.039

13 Z. Wu, W. Fu, R. Xue, and W. Wen: Information 7 (2016) 39. https://doi.org/10.3390/info7030039

14 B. Tang, Z. Zhu, and J. Luo: Assembly Autom. 37 (2017) 114. https://doi.org/10.1108/AA-03-2016-024

15 W. Zhang, H. Lu, M. Ma, C. Kong, and S. Wang: 2019 6th Int. Conf. Systems and Informatics (ICSAI, Shanghai, China) (2019).

16 Y. Li, X. Yao, and J. Zhou: J. Mech. Eng. 62 (2016) 577. https://doi.org/10.5545/sv-jme.2016.3545

17 X. Zhou: J. Phys. Conf. Series 1533 (2020) 022010. https://doi.org/10.1088/1742-6596/1533/2/022010

18 O. Montiel, U. Orozco-Rosas, and R. Sepúlveda: Expert Syst. Appl. 42 (2015) 5177. https://doi.org/10.1016/j. eswa.2015.02.033

19 A. Tuncer and M. Yildirim: Comput. Electr. Eng. 38 (2012) 1564. https://doi.org/10.1016/j. compeleceng.2012.06.016

20 H. Zhu and Y. Peng: Procedia Comput. Sci. 166 (2020) 557. https://doi.org/10.1016/j.procs.2020.02.022

21 P. K. Das, H. S. Behera, P. K. Jena, and B. K. Panigrah: J. Electr. Syst. Inf. Technol. 3 (2016) 295. https://doi. org/10.1016/j.jesit.2015.12.003

22 X. Zhang, Y. Zhao, N. Deng, and K. Guo: Int. J. Adv. Rob. Syst. 13 (2016) 91. https://doi.org/10.5772/63484

23 Y. Li, R. Cui, D. Xu, and S. Liu: 2018 3rd Int. Conf. Advanced Robotics and Mechatronics (ICARM, Singapore) (2018).

24 J. Liao, S. Lima, and A. Rocha: J. Intell. Fuzzy Syst. 35 (2018) 2821. https://doi.org/10.3233/jifs-169635

25 M. S. Alajmi and A. M. Almeshal: Materials 13 (2020) 2986. https://doi.org/10.3390/ma13132986

\section{About the Author}

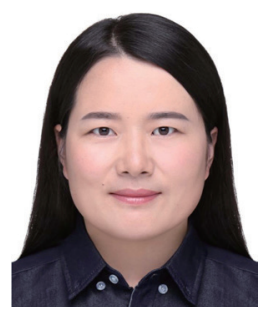

Jing Song received her B.S. degree from Zhengzhou University of Light Industry and her M.S. and Ph.D. degrees from Guangdong University of Technology, China, in 2000, 2006, and 2012, respectively. She was a visiting scholar at South China University of Technology from 2015 to 2016 and at California State University, Chico, USA, from 2018 to 2019. She joined College of Port and Shipping Management, Guangzhou Maritime University, China in 2014, where she is currently a lecturer. Her research interests include logistics system engineering and supply chain management. (84864809@qq.com) 